\title{
Diagnostic utility of inflammatory markers in septic arthritis in pediatric patients in atertiary care hospital in Bangalore
}

\author{
Manasa.S. ${ }^{1}$, Mahantesh S. ${ }^{2}$ \\ ${ }^{1}$ Dr.Manasa.S, Scientist B., ${ }^{2}$ Dr. Mahantesh S, Associate Professor; both authors are affiliated with Department of \\ Microbiology, IndiraGandhi Institute of Child Health, Bangalore, India.
}

Corresponding Author: Dr. Mahantesh. S, Associate Professor, Department of Microbiology, IGICH Bangalore, India. E-mail:manasabharadwaj86@gmail.com

\begin{abstract}
Objectives: Septic arthritis is a disease associated with serious morbidity to the patient. The causes of monoarticular arthritis are numerous, with septic arthritis being the most important entity. The diagnosis is rarely established by the history and physical examination, and the clinician is led to rely on ancillary tests.As there are very limited studies relating to the usefulness of CRP, synovial fluid WBCand ESR in diagnosing septic arthritis in the pediatric population, this study was donein pediatric population in a tertiary care hospital in Bangalore. Materials and Methods: This is a retrospective study done over a period of 24months from January 2017 to December 2018. The study population included all the suspected patients of septic arthritis. Their inflammatory markers like WBC count, ESR and CRP were compared with the culture of the synovial fluid. Results: Our records search identified 335 potential cases. 134 cases fulfilled the diagnostic criteria for septic arthritis out of the 335 included in the study. Sensitivity of ESR was: $98 \%$ using a cutoff of $\geq 10 \mathrm{~mm} / \mathrm{h}$ and $94 \%$ using a cutoff of $\geq 15 \mathrm{~mm} / \mathrm{h}$. The sensitivity of CRP was $92 \%$ using a cutoff of $\geq 20 \mathrm{mg} / \mathrm{L}$. synovial fluid sample with WBC of $>50000 / \mu \mathrm{L}$ of which $>75 \%$ are polymorphonuclear cells is considered to suggest Septic arthritis. Conclusion: Using our data and comparing it with published data we propose that it is unnecessary to perform further investigation for septic arthritis in patients who have all three of: $\mathrm{ESR}$ value $<15 \mathrm{~mm} / \mathrm{h}, \mathrm{WBC}<50,000 / \mu \mathrm{L}$ and CRP value $<20 \mathrm{mg} / 1$.
\end{abstract}

Key words: Septic arthritis; C reactive protein (CRP), ESR, Synovial fluid WBC

\section{Introduction}

Septic arthritis as joint sepsis caused by pathogenic inoculation of the joint by direct or haematogenous routes, rather than an immunological response to pathogens such as that seen in reactive arthritis. Delayed or inadequate treatment of septic arthritis can lead to irreversible joint destruction with subsequent disability, and in addition there is significant mortality with an estimated case fatality rate of $11 \%$. It is therefore vital that the diagnosis is made rapidly and that treatment is started promptly.One of the difficulties surrounding the assessment of joint infection is that patients often present to clinicians who are inexperienced in the management of musculoskeletal disease. Prognosis is optimized when the diagnosis is made quickly and appropriate treatment is given. Even when management is correct, a significant number of cases result in irreversible joint damage and, in some patients, overwhelming septicaemia.

Manuscript received: $16^{\text {th }}$ May 2019

Reviewed: $26^{\text {th }}$ May 2019

Author Corrected: 02 $2^{\text {nd }}$ June 2019

Accepted for Publication: $07^{\text {th }}$ June 2019
The purpose of our study was to examine the diagnostic utility of CRP in patients with septic arthritis.

\section{Materials and Methods}

Source of data: This is a retrospective study done over a period of 24months from January 2017 to December 2018. The study will be conducted in the department of Microbiology, IndiraGandhi Institute of Child Health. The study population included All the suspected septic arthritis patientsin pediatric patients. Their inflammatory markers like ESR, CRP and WCC were noted. Their culture reports were compared.

Inclusion criteria- All the suspected septic arthritisin pediatric patients were included in the study

Exclusion Criteria: Patients above 18yrs were excludedand alsoPatients who had "dry taps" were excluded from the study. 
Methodology: We retrospectively reviewed all cases of children who were evaluated for septic arthritis and presenting with acute limp unrelated to trauma. Cases were divided into two diagnostic groups: septic arthritis and non-septic arthritis. Diagnoses were defined on the basis of the results of cultures of joint fluid and blood,

\section{Original Research Article}

and the clinical course. Univarate analysis was performed to evaluate the predictive value of previously identified factors in determining septic arthritis: WCC $>12.0$, erythrocyte sedimentation rate $(\mathrm{ESR})>40, \mathrm{C}$ reactive protein $(\mathrm{CRP})>20$.

\section{Results}

The medical charts were reviewed for demographic (age, sex), clinical (operative findings, diagnoses) and laboratory data of WCC, ESR and CRP were also collected. Our records search identified 335 potential cases. 134 cases fulfilled the diagnostic criteria for septic arthritis out of the 335 cases included in the study. In the134 proved septic arthritis cases 92 $(68.6 \%)$ were male babies and $42(31.3 \%)$ were female babies. Out of 201 Non septic arthritis cases 161 (80.1\%) were males and $40(19.9 \%)$ were females.

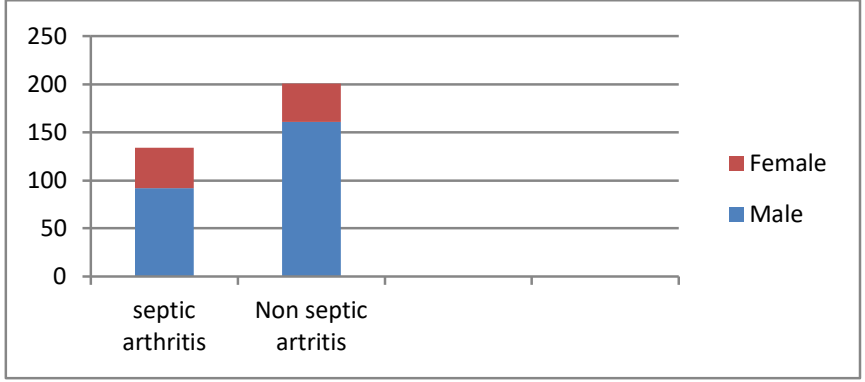

Graph-1: Distribution of sex in septic arthritis and non septic arthritis

The Mean age of the enrolled cases is 6.2years. Sensitivity of inflammatory markers in diagnosing septic arthritis as follows: ESR was: $98 \%$ using a cutoff of $\geq 10 \mathrm{~mm} / \mathrm{h}$ and $94 \%$ using a cutoff of $\geq 15 \mathrm{~mm} / \mathrm{h}$. The sensitivity of CRP was $92 \%$ using a cutoff of $\geq 20 \mathrm{mg} / \mathrm{L}$. synovial fluid sample with $\mathrm{WBC}$ of $>50000 / \mu \mathrm{L}$ of which $>75 \%$ are polymorphonuclear cells is considered to suggest Septic arthritis.

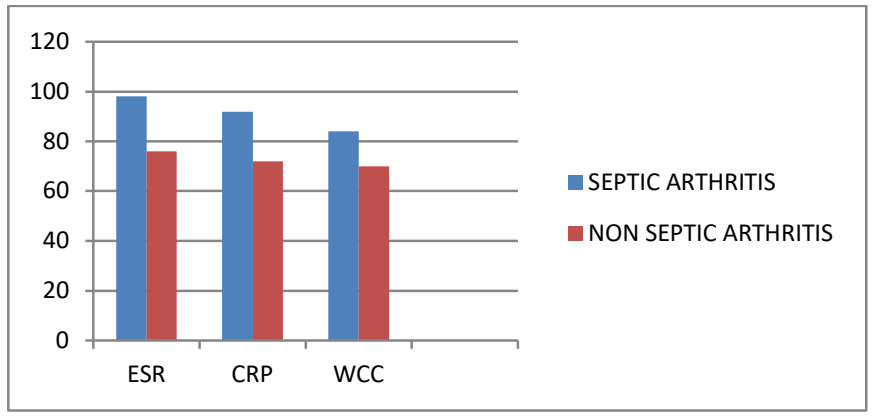

Graph-2: Percentage of sensitivity of the inflammatory markers in Diagnosing Septic and Non septic arthritis

\section{Discussion}

Acute monoarticular arthritis in presenting to the emergency department (ED) has multiple potential etiologies including infection (bacterial, fungal, mycobacterial, viral), crystalloid arthropathies, rheumatoid arthritis, lupus, and trauma $[1,-3]$. Septic (i.e., bacterial) arthritis has an annual incidence of 10 per 100,000 individuals in the United States and is more common among those with rheumatoid arthritis or a prosthetic joint, with up to 70 cases per 100,000 [4]. Patients with human immunodeficiency virus (HIV) are also at increased risk for nongonococcal septic arthritis [5].
Septic arthritis most commonly affects the knee, which accounts for approximately $50 \%$ of cases. In decreasing order of frequency, septic arthritis also affects the hip, shoulder, and elbow, although virtually any articular surface can become infected [6]. Most cases result from hematogenous spread, since bacterial organisms can easily enter the synovial fluid because synovial tissue lacks a basement membrane. Prompt diagnosis to facilitate appropriate antibiotic management of septic arthritis is essential, since cartilage can be destroyed within days, and in-hospital mortality of treated infections can be as high as $15 \%$ [7]. 
Permanent disability and increased mortality are associated with delayed presentations and diagnosis [79]. Prior research suggests that using history, physical examination, and synovial tests, clinicians are able to deduce the etiology of acute nontraumatic monoarticular arthritis within 3 days in most cases [10].

Since emergency physicians often lack the luxury of 3day admissions for most monoarticular arthritis patients, identification of key diagnostic findings to accurately differentiate septic from nonseptic arthritis within minutes to hours is essential.

The predominant causative pathogens in septic arthritis are Staphylococcus aureus and Streptococcus, accounting for up to $91 \%$ of cases $[11,12]$. In the elderly, the immunocompromised and in those patients who have had intravascular devices or urinary catheters inserted, infection with a Gram-negative enteric bacillus is more common. Due to a combination of factors the aetiology of septic arthritis is changing. The increasing incidence of surgical arthroplasty provides a prosthetic environment where coagulase-negative staphylococci, which are unusual pathogens in native joint sepsis, are able to flourish.

This often establishes low-grade infection and subsequent prosthesis failure. It is a matter of concern that the ability of organisms to develop antibiotic resistance is highlighted by the recent emergence of communityassociated methicillin-resistant S. aureus (CA-MRSA) in patients who do not have traditional risk factors for MRSA acquisition. CA-MRSA has been responsible for cases of musculoskeletal sepsis in both North America (USA) and the United Kingdom (UK) and requires alternative antimicrobial strategies to the more common healthcareassociated MRSA $[12,13]$.

In addition, the increase in both iatrogenic immunosuppression and HIV infection means that more unusual organisms such as mycobacteria are increasing in incidence [14-15]. Septic arthritis continues to cause significant morbidity and mortality despite adequate removal of purulent material and prompt, appropriate antibiotic therapy. One fruitful area of current research addresses the concept that successful treatment requires not only the elimination of pathogenic bacteria but also the downregulation of the heightened immune response that appears to hinder, rather than help, the host's defence mechanisms. There is a common misconception that septic arthritis affects one joint only, but evidence suggests that in up to $22 \%$ of cases the presentation is polyarticular. Large joints are more commonly affected than small joints and in up to $60 \%$ of cases the hip or the knee is involved.

\section{Original Research Article}

It is very important to diagnose septic arthritis clinically before the confirmation is obtained by culture results are obtained of the joint fluid and blood. The inflammatory markers used for the diagnosis of the septic arthritis are ESR, CRPand WCC.

ESR measures the distance through which erythrocytes fall within 1 hour in a vertical tube of anticoagulated blood [16]. ESR and its significance was first reported by Dr. Edmund Faustyn Biernacki in 1897 [17]. He observed that the rate at which blood settled varied among different individuals, that blood with smaller amounts of erythrocytes settled more quickly, and that the rate of settling depended on plasma fibrinogen levels. He also noted that ESR was high in patients with febrile diseases associated with high fibrinogen levels (eg, rheumatic fever), whereas it was low in defibrinated blood.

In 1918, Swedish hematologist Robert Sanno Fåhraeus presented the results of his analyses of the differences in erythrocyte sedimentation rates in pregnant and nonpregnant women, seeing the test as a possible indicator of pregnancy [17]. In 1921, Dr. Alf Vilhelm Albertsson Westergren, a Swedish internist, published his observations of erythrocyte sedimentation in patients with pulmonary tuberculosis [17]. Westergren defined standards for the performance of the ESR test, and the Westergren method of measuring ESR is still widely used today. In modern medicine, the ESR test is sometimes referred to as the Fåhraeus-Westergren test[18].

Methods of Measuring ESR- In the Westergren method, a fixed amount of blood is drawn into a vertical tube anticoagulated with sodium citrate. The blood is left to settle for 1 hour, after which the distance between the top of the blood column and the top layer of the red blood cells (RBCs) below is measured. The ESR is thus reported in millimeters/hour. Newer methods employ a special centrifuge and automated machines and can yield results in as quickly as 5 minutes[18,19].

Physiology of ESR- Erythrocyte aggregation is influenced by the surface charge of red cells and the dielectric constant of the surrounding plasma, with the latter depending on the concentration and symmetry of plasma proteins. The negatively charged erythrocytes tend to repel one another, but in the presence of positively charged large asymmetric proteins, erythrocyte aggregation and rouleaux formation are promoted[20, 21]. Erythrocyte aggregates fall faster, thereby increasing the ESR [20]. Fibrinogen, one of the major acute phase reactants and a highly asymmetric protein, has the greatest effect on ESR. 
Immunoglobulins in high concentrations also increase erythrocyte aggregation. Immunoglobulin $\mathrm{G}(\mathrm{IgG})$ is the most abundant of the immunoglobulins with the highest rate of synthesis, and it has a half-life ranging from 7 to 21 days depending on the subclass [22]. Fibrinogen has a half-life of approximately 100 hours [23]. Because fibrinogen and immunoglobulins are two of the major proteins affecting ESR, and because both have relatively long half-lives, ESR remains elevated for days to weeks after resolution of inflammation[19].

False Results- Non-inflammatory factors can also affect ESR. Erythrocyte shape and size as well as blood viscosity affect erythrocyte aggregation. With a reduced hematocrit (ie, anemia), the upward flow of plasma increases and erythrocyte aggregates fall faster, increasing ESR. With polycythemia, crowding of erythrocytes decreases the compactness of the rouleaux, slowing ESR [16]. Sickled and anisocytotic RBCs have a decreased tendency to form rouleaux, so ESR decreases in these states as well [16-19].

Because erythrocyte aggregation is facilitated by the presence of high molecular weight proteins, patients with hypo- or afibrinogenemia as well as those with hypo- or agammaglobulinemia can have a falsely low ESR in the face of active inflammation. Similarly, IVIg therapy, despite its anti-inflammatory effects, typically increases the ESR due to the increase in serum $\mathrm{IgG}$, as happens in Kawasaki disease patients after treatment with IVIg [24,25].

C-Reactive Protein- CRP was first discovered in 1930 by Tillet and Francis during their serologic studies of patients with pneumococcal pneumonia. They observed precipitation in the serum of sick patients, noting that precipitation decreased as patients recovered. They determined that precipitation occurred due to a protein in the serum that reacted with the C-polysaccharide of pneumococcal cell walls, hence the name "C $\mathrm{C}$-reactive protein."[26].

Methods of Measuring CRP- CRP was originally measured via the Quellung reaction, wherein precipitation of the C-polysaccharide in the serum was determined [27]. Before quantitative methods were developed, CRP was reported merely as being either "present" or "absent."[21].

Eventually, more precise quantitative methods were developed, of which the most commonly used today is nephelometry. With this technique, light scattering from CRP-specific antibody aggregates is measured, yielding results in as little as 15 to 30 minutes [28]. Today, highsensitivity assays are being utilized to detect even low

\section{Original Research Article}

levels of CRP, which helps to determine cardiovascular risk, particularly in the adult population [29].

Physiology of CRP- Synthesis of CRP occurs in the liver and is stimulated by the presence of cytokines, particularly interleukin (IL)-1 beta, IL-6, and tumor necrosis factor (TNF). It increases within 4 to 6 hours after the onset of inflammation or injury, doubling every 8 hours and peaking at 36 to 50 hours. Because of the short half-life (4-7 hours), plasma concentration depends only on the rate of synthesis; CRP levels thus drop quickly after inflammation resolves [28].

False Results- CRP is synthesized by the liver; therefore, hepatic failure may impair CRP production. In a small study by Silvestre et al [30]. CRP levels were found to be markedly low despite overwhelming sepsis in patients with fulminant hepatic failure. The authors proposed that in patients with fulminant hepatic failure, CRP should be used more as a marker of liver dysfunction rather than of infection [30].

Differences Between ESR and CRP- CRP levels fall more quickly than the ESR, normalizing 3 to 7 days after resolution of tissue injury, whereas ESR can take up to weeks to normalize. Therefore, it is appropriate to use CRP for monitoring "acute" disease activity such as acute infection (eg, pneumonia, orbital cellulitis). In contrast, measurement of ESR is beneficial in the monitoring of chronic inflammatory conditions such as systemic lupus erythematosus or inflammatory bowel diseases. As discussed above, many factors can falsely increase or decrease ESR, whereas CRP is less likely to be affected (except in cases of liver failure). ESR requires a fresh specimen of whole blood, whereas CRP can be measured from stored specimens of serum or plasma. CRP changes minimally with age, whereas ESR rises with age and is generally higher in females.

In our study the sensitivity of the inflammatory markers like CRP, ESR and WCC were calculated using standard statistical procedure.Sensitivity of ESR was $98 \%$ using a cutoff of $\geq 10 \mathrm{~mm} / \mathrm{h}$ and $94 \%$ using a cutoff of $\geq 15 \mathrm{~mm} / \mathrm{h}$. The sensitivity of CRP was $92 \%$ using a cutoff of $\geq 20 \mathrm{mg} / \mathrm{L}$.synovial fluid sample with WBC of $>50000 / \mu \mathrm{L}$ of which $>75 \%$ are polymorphonuclear cells is considered to suggest Septic arthritis.

\section{Conclusion}

On the basis of these findings, it can be safely hypothesized that Distinguishing between transient synovitis and septic arthritis is essential as the management and potential complications are very different. 
Our study provides strong support for the use of nonweight bearing status, fever $\left(>37.5^{\circ} \mathrm{C}\right)$, WBC $>50,000 / \mu \mathrm{L}, \mathrm{ESR}>40 \mathrm{~mm} / \mathrm{h}, \mathrm{CRP}>20 \mathrm{mg} / \mathrm{L}$ in the differentiation of the conditions. Using our data and comparing it with published data we propose that it is unnecessary to perform further investigation for septic arthritis in patients who have all three of: ESR value $<40$, WCC $<12$ and CRP value $<20$.

Findings: Nil; Conflict of Interest: None initiated Permission from IRB: Yes

\section{References}

1. Goldenberg DL. Septic arthritis. Lancet. 1998 Jan 17;351(9097):197-202.

2. Margaretten ME, Kohlwes J, Moore D, et al. Does this adult patient have septic arthritis? JAMA. 2007 Apr 4;297(13):1478-88. DOI:10.1001/jama.297.13.1478

3. Till SH, Snaith ML. Assessment, investigation, and management of acute monoarthritis. J AccidEmerg Med. 1999 Sep;16(5):355-61.

4. Kaandorp CJ, Van Schaardenburg D, Krijnen P, et al. Risk factors for septic arthritis in patients with joint disease. A prospective study. Arthritis Rheum. 1995 Dec;38(12):1819-25.

5. Saraux A, Taelman H, Blanche $P$, et al. HIV infection as a risk factor for septic arthritis. Br J Rheumatol. 1997 Mar;36(3):333-7.

6. Kaandorp CJ, Dinant HJ, van de Laar MA, et al. Incidence and sources of native and prosthetic joint infection: a community based prospective survey. Ann Rheum Dis. 1997 Aug;56(8):470-5.

7. Gupta MN, Sturrock RD, Field M. A prospective 2year study of 75 patients with adult-onset septic arthritis. Rheumatology (Oxford). 2001 Jan;40(1):2430 .

8. Cooper C, Cawley MI. Bacterial arthritis in an English health district: a 10 year review. Ann Rheum Dis. 1986 Jun;45(6):458-63. DOI:10.1136/ard.45.6.458

9. Söderquist B, Jones I, Fredlund H, et al. Bacterial or crystal-associated arthritis? Discriminating ability of serum inflammatory markers. Scand J Infect Dis. 1998;30(6):591-6.

10. Freed JF, Nies KM, Boyer RS, et al. Acute monoarticular arthritis. A diagnostic approach. JAMA. 1980 Jun 13;243(22):2314-6.

\section{Original Research Article}

11. Weston VC, Jones AC, Bradbury N, et al. Clinical features and outcome of septic arthritis in a single UK Health District 1982-1991. Ann Rheum Dis. 1999 Apr;58(4):214-9.

12. Gupta MN, Sturrock RD, Field M. A prospective 2year study of 75 patients with adult-onset septic arthritis. Rheumatology (Oxford). 2001 Jan;40(1):2430. DOI:10.1093/rheumatology/40.1.24

13. Kroesen S, Widmer AF, Tyndall A, et al. Serious bacterial infections in patients with rheumatoid arthritis under anti-TNF- alpha therapy. Rheumatology (Oxford). 2003 May; 42(5): 617-21. DOI:10.1093/ rheumatology/ keg263

14. Murdoch DM, McDonald JR. Mycobacterium avium-intracellulare cellulitis occurring with septic arthritis after joint injection: a case report. BMC Infect Dis. 2007 Feb 26;7:9. DOI:10.1186/1471-2334-7-9

15. Zalavras CG, Dellamaggiora R, Patzakis MJ, et al. Septic arthritis in patients with human immunodeficiency virus. Clin Orthop Relat Res. 2006 Oct; 451:46-9. DOI:10.1097/ 01.blo. 0000229305. 97888.17

16. Brigden ML. Clinical utility of the erythrocyte sedimentation rate. Am Fam Physician. 1999 Oct 1;60(5):1443-50.

17. Grzybowski A, Sak JJ. Who discovered the erythrocyte sedimentation rate? J Rheumatol. 2011 Jul;38(7): 1521-2; author reply 1523. doi: 10.3899 /jrheum. 101312.

18. Janson LW, Tischler M. The Big Picture: Medical Biochemistry. New York, NY: McGraw-Hill; 2012.

19. Batlivala SP. Focus on diagnosis: the erythrocyte sedimentation rate and the C-reactive protein test. Pediatr Rev. 2009 Feb;30(2):72-4. doi: 10.1542/pir.30$2-72$.

20. Husain TM, Kim DH, C-reactive protein and erythrocyte sedimentation rate in orthopaedics. Univ Pennsylvania Orthop J. 2002;15:13-16.

21.Wilson DA. Immunologic tests. In: Walker HK, Hall WD, Hurst JW, eds. Clinical Methods: The History, Physical, and Laboratory Examinations. 3rd ed. Boston, MA: Butterworth-Heineman; 1990.

22. Isenman DE, Mandle R, Carroll MC. Complement and immunoglobulin biology. In: Hoffman R, Benz EJ, Silberstein LE, Heslop HE, Weitz JI, Anastasi J, eds. Hematology: Basic Principles and Practice. 6th ed. Philadelphia, PA: Elsevier Saunders; 2013:228-246.e4. 
23. Kamath S, Lip GY. Fibrinogen: biochemistry, epidemiology and determinants. QJM. 2003 Oct;96(10): 711-29. DOI:10.1093/qjmed/hcg129

24. Nimmerjahn F, Ravetch JV. The anti-inflammatory activity of IgG: the intravenous IgG paradox. J Exp Med. 2007 Jan 22; 204(1):11-5. Epub 2007 Jan 16.

25. Salehzadeh F, Noshin A, Jahangiri S. IVIG Effects on Erythrocyte Sedimentation Rate in Children. Int J Pediatr. 2014;2014:981465. doi: 10.1155/2014/981465. Epub 2014 Jan 29.

26. Vermeire S, Van Assche G, Rutgeerts P. C-reactive protein as a marker for inflammatory bowel disease. Inflamm Bowel Dis. 2004;10 (5):661-665. doi:10.1097/00054725-200409000-00026

\section{Original Research Article}

27. Nudelman R, Kagan BM. C-reactive protein in pediatrics. Adv Pediatr. 1983;30:517-47.

28. Jaye DL, Waites KB. Clinical applications of Creactive protein in pediatrics. Pediatr Infect Dis $\mathrm{J}$. 1997;16(8):735-746; quiz 746-747. doi:10.1097/ 00006454- 199708000-00003

29. Koenig W. High-sensitivity C-reactive protein and atherosclerotic disease: from improved risk prediction to risk-guided therapy. Int $\mathrm{J}$ Cardiol. 2013 Oct 15;168(6):5126-34. doi: 10.1016/j.ijcard.2013.07.113. Epub 2013 Aug 24

30. Silvestre JP, Coelho LM, Póvoa PM. Impact of fulminant hepatic failure in C-reactive protein? $\mathrm{J}$ Crit Care. 2010 Dec;25 (4): 657.e7-12. doi: 10.1016/j. jcrc.2010.02.004. Epub 2010 Apr 8.

\section{How to cite this article?}

Manasa.S., Mahantesh S. Diagnostic utility of inflammatory markers in septic arthritis in pediatric patients in atertiary care hospital in Bangalore. Trop J Path Micro 2019;5(6):343-348. doi:10.17511/jopm.2019.i06.02. 